\title{
A Suspected Supernova in A.D. II8I
}

\author{
F.R.Stephenson \\ (Department of Geophysics and Planetary Physics, School of Physics, \\ University of Newcastle upon Tyne)
}

\section{SUMMARY}

The new star which appeared in Cassiopeia in A.D. II8I attracted considerable attention in the Far East. It was visible for about six months, and its position was carefully recorded by the Chinese and Japanese astronomers. Their observational evidence favours the identification of the star as a supernova of type I. It is proposed that the radio source ${ }_{3} \mathrm{C} 58$ is the remnant of the new star.

\section{OBSERVATIONS OF THE STAR}

The 'guest star' which appeared in A.D. II8I probably aroused more interest than any other new star which was seen before modern times, with the exception of the brilliant supernovae of A.D. 1006, I054, I572 and 1604. The star was observed independently in north and south China (the Chin and Sung empires) and also in Japan. As well as being mentioned in several Japanese histories, the guest star was reported in the diaries of certain courtiers living at the time.

The original Chinese and Japanese records in the references cited by Kanda Shigeru (1935), by Hsi Tsê-tsung (1955), by Ho Peng Yoke (1962) and by Hsi \& Po Shu-jen (1965) have been consulted by the author. Translations of these are as follows.

(a) Sung Shih ('History of the Sung Dynasty'; written c. 1345) $(56 / 25 \mathrm{~b})$. 'On the day chi-szú in the sixth month of the eighth year of the Ch'un-hsi reign period [II8I August 6] a guest star ( $k$ 'o hsing) appeared in $K^{\prime}$ 'uei hsiu and invading (fan) Ch'uan-shê until the day $k u e i-y u$ of the first month of the following year [I182 February 6], altogether 185 days; only then was it extinguished (mieh)'.

(b) Chin Shih ('History of the Chin (Kin) Dynasty'; written c. 1345) (20/129). 'On the day chia-wu in the sixth month of the 21st year of the Ta-ting reign period [II8I August II] a guest star was seen at (yü) Hua-kai altogether for 156 days; then it was extinguished'.

(c) Dainihonshi ('History of Great Japan'; written I715). (c. 359). 'On the day keng-wu, the 25th day of the sixth month of the first year of the Yowa reign period [I 18I August 7] a guest star appeared at the North near (chin) Wang-liang and guarding (shou) Ch'uan-shê'. 
In the following Japanese descriptions I have omitted the date when it is identical with that in the Dainihonshi record. Titles of the various works, which are often completely irrelevant, are not translated. I am grateful to Dr M.I.Scott, of the University Library, Cambridge, for supplying the notes shown in parentheses.

(d) Meigetsuki (Diary of Fujiwara Sadaie [1180-I235], a poetcourtier).

Following the appearance of a guest star on the 28th day of the roth month of the 2nd year of the Kanki reign period [1230 December 4], Fujiwara recorded numerous observations of early guest stars in his diary. The star of II8I is reported in the entry for the 8th day of the IIth month of the 2nd year of the Kanki reign period [1230 December 13]. The account is identical with that in the Dainihonshi.

(e) Gyokuyo (Diary of Fujiwara Kanezane [II64-I200], courtier of the imperial court).

'On the 28th day (commentary: $=k u e i-y u$ ) it was heard that since the 25th day a guest star had been present in the inner sky (commentary: beside Wang-liang), a sign of abnormality indicating that at any moment we can expect control of the administration to be lost'.

(f) Hyakurensho (History of the imperial court from 967 to 1259 . Written c. 1260).

'A guest star was seen at the north pole'.

(g) Azuma Kagami (History of the Kamakura military government [1180-1266]. In two parts-mid I3th and mid I4th century). The NASA translation of the paper by Hsi \& Po calls this work the Wagazuma Kagami while that by Yang refers to it as the Matsuyama Kagami. Both of these renderings are incorrect.

'At the hour $h s \ddot{u}$ ( $=19^{\mathrm{h}}$ to $2 \mathrm{I}^{\mathrm{h}}$ local time) a guest star was seen in the north-east. It was like Saturn and its colour was bluish-red and it had rays. There had been no other example since that appearing in the 3rd year of Kankō [A.D. 1006]'.

The record appears to be incomplete. The NASA translation prefers 'it was as large as Saturn', while Shinoda (1960) renders the passage 'it was the colour of Saturn'. The translation by Yang which reads 'it was as big as Tsung Hsing [I IO, I I I Herculis]' is quite erroneous.

(h) Kikki (Diary of Yoshida Tsunefusa [I 140-1200], a courtier of the imperial court.)

'The twenty-fifth day keng-shen of the eighth month [II8I September 26]. Today was the occasion for making auspicious offerings for a good harvest. A guest star had been seen in the NNE and at the present time it still has not faded away.' 
Shklovsky (1960) quotes an undisclosed oriental source which states that the guest star was as bright as Jupiter. I can find no record of this. The star does not seem to have attracted attention in Korea. The Koryó-sa ('History of the Kingdom of Koryō') contains detailed astronomical records for this period (12th year of Myongjong) but the guest star is overlooked. Guest star records from Korea are rather fragmentary; there are no references to the supernovae of 1006 and 1054 in Korean history.

\section{STELLAR NATURE OF THE GUEST STAR}

The term k'o hsing ('guest star' or 'visiting star') was used throughout the far east to describe a temporary star when the descriptions hui hsing ('broom star') or po hsing ('rayed star') were not appropriate. Both hui hsing and po hsing normally refer to comets, the former when a long tail is present, the latter when the tail is either short or absent. There is a separate expression for a meteor (liu hsing - 'flowing star'). The usual interpretation of $k^{\prime} o$ hsing is thus a nova or supernova, but records of moving guest stars are not infrequent, e.g. in A.D. 990, I005, I02I and I273 (Ho 1962, p. 137). This implies that occasionally it proved difficult to distinguish between a nova and a comet with a short or non-existent tail.

The star observed in China in A.D. I3I5 was described first as a guest star while a month later it became a broom star. The star of I 66 was called a guest star in China and a broom star in Japan. However, when a temporary star remained visible for a lengthy period there was apparently little confusion. In the extensive catalogue of comets and novae observed in the Far East prepared by Ho (op. cit.), stars other than guest stars were rarely observed for more than about two months. The rayed star of A.D. 277 was seen for six months, while broom stars were seen for about four months in A.D. 1053, I204, I458 and 1532. All were correctly described as comets, for in every case a detailed account of the motion is given.

Two exceptional records refer to stars seen in A.D. 683 and 1572. A broom star was discovered in Auriga by the Chinese in April of A.D. 683. Another broom star was seen in the same part of the sky by the Koreans six months later. After the sighting in China observation of Auriga would be interrupted owing to the conjunction with the Sun. Hsi \& Po suggest that the Chinese and Korean accounts refer to a single supernova and they propose an identification with the powerful radio source Auriga $A$. This could only be verified if the radio source fits the age well, for the position is vague and both records describe the star as a broom star. The supernova of 1572 (Tycho's supernova) 
was called a broom star in the draft version of the Ming Shih ('History of the Ming Dynasty') but was correctly called a guest star in the official history and also in Korea.

Certain unidentified 'stars' apparently could not be classified in any of the usual categories, e.g. in A.D. I388, I404, I430 and I49I. However, as all were of short duration, they need not concern us here.

Records of guest stars appearing for several months, or even more than a year, are fairly numerous, but of these only in one instance (A.D. 902-see Section 3) is any motion suggested. None of the descriptions of the new star of II8I contains any hint of motion, and all use the term $k^{\prime} o$ hsing. The general character of the two Chinese records (a) and (b) indicates that the guest star remained fixed for the I85 or I56 days of observation. This is also true of the Kikki record (h). The remaining Japanese accounts, with the exception of the Gyokuyo record (e), report only the first sighting, but this feature is characteristic of almost all Japanese observations of guest stars. The use of the expression shou ('guarding') in the Dainihonshi (c) and Meigetsuki (d) accounts, however, implies that the star remained fixed. This term was defined in various ways, e.g. 'to attach and stay by the side of (a constellation or another celestial body)' or 'to stay (in a particular place) without leaving' (Ho I966, p. 37).

In Section 10 it is shown that the guest star of 1181 appeared at a very low galactic latitude (between 0 and $+4 \mathrm{deg}$.); this is characteristic of novae, and especially of supernovae. This result gives support to the evidence discussed above which seems to establish that the star was of a stellar rather than cometary nature.

\section{TYPE OF OUTBURST}

The oriental asterisms mentioned in the accounts (a), (b), (c) and (d) in Section I are all in the vicinity of Cassiopeia. This location is confirmed by the Azuma Kagami record (g) which states that the guest star appeared on August 7 between $19^{\mathrm{h}}$ and $2 \mathrm{I}^{\mathrm{h}}$ (local time) in the north-east. As the Sun was then at R.A. $9^{\mathrm{b}} 30^{\mathrm{m}}$, the star (sighted in China the night before) would probably be observed soon after $19^{\mathrm{h}} 30^{\mathrm{m}}$ local time, when darkness fell. By this time Cassiopeia (R.A. about $0^{\text {h }}$ ) would be considerably to the east of the pole. The observation quoted in the Hyakurensho (f)-'at the north pole'-gives only a general indication of position.

Hsi, who regarded the guest star as a supernova, deduced an approximate position R.A. $\mathrm{I}^{\mathrm{h}} 4 \mathrm{0}^{\mathrm{m}}$, Dec. $+70^{\circ}$ (galactic co-ordinates $l^{\mathrm{II}}=127^{\circ}, b^{\mathrm{II}}=+9^{\circ}$ ) at $1900 \cdot 0$. This led Shklovsky to suggest a 
possible identification of the star with the powerful radio source Cassiopeia A, a known supernova remnant. Hsi \& Po gave a revised position (R.A. $\mathrm{I}^{\mathrm{h}} 30^{\mathrm{m}}$, Dec. $+65^{\circ} ; l^{\mathrm{II}}=\mathrm{I} 27^{\circ}, b^{\mathrm{II}}=+3^{\circ}$ ). They regarded the guest star as a supernova, but were not able to associate it with any known SNR.

It is well known that ordinary novae, unlike supernovae, are not associated with radio sources. The quantity of matter ejected in a nova explosion is extremely small-about $1 \mathrm{I}^{-4}$ of the mass of the star. In $2 \mathrm{C}$, the second Cambridge catalogue of radio sources (Shakeshaft et al. 1955), it was suggested that the source $2 \mathrm{C}_{1595}$ was possibly identifiable with Nova Aquilae, which appeared in 1918. This nova reached an apparent magnitude of $-I \cdot I$ at maximum, only about a magnitude fainter than Kepler's supernova of 1604 . The improved positional accuracy of $3 \mathrm{C}$ (Edge et al. 1959) made it clear that $2 \mathrm{C}$ I595 $(=3 \mathrm{C} 39 \mathrm{r})$ had no connection with Nova Aquilae. None of the many faint radio sources catalogued in $4 \mathrm{C}$ (Gower et al. 1967) can be associated with this or any other nova. The expanding shell of nebulosity from a nova explosion dissipates in a few decades. In consequence, the only remnant observable at the present time of an early guest star which was an ordinary nova would be the usual Oe-type star about 12 magnitudes fainter than the maximum.

The evidence that the guest star of II8I was a supernova rather than an ordinary nova is based mainly on the extensive period of observation and low galactic latitude. The light curve of a typical nova shows a much more rapid rise and decline than that of a supernova (regardless of type), while the concentration towards the galactic equator of novae which are bright enough to attract the attention of the unaided eye is not nearly so marked as for the much more distant supernovae. Occasionally, however, slow bright novae do appear in low galactic latitude. The possibility exists of an early record of such an occurrence being interpreted as an account of a supernova explosion. It is thus important to examine the observations of the bright novae which have appeared in recent times.

The period from 1901 to 1942 was remarkable for the number of bright novae. Five, appearing in 190I, 1918, 1925, 1934 and 1942, reached an apparent visual magnitude brighter than +2 . From before A.D. I 700 until I90I no comparable occurrences were observedexcluding $\eta$ Car which has shown remarkable variation since 1729 and may have been a subluminous supernova (Payne-Gaposchkin I957, p. 97; Zwicky 1965). No bright novae have appeared since 1942. Brief details for the five principal novae of the present century are given in Table I. The source is table I'I of the work by Payne-Gaposchkin. We might expect similar stars to have attracted attention in the Far East in ancient and medieval times. 
TABLE I

The bright novae of the present century

$\begin{array}{llcc}\text { Year } & \text { Constellation } & \text { Mag. } & b^{\text {II }} \\ \text { I901 } & \text { Perseus } & +0 \cdot 2 & -9^{\circ} \\ 1918 & \text { Aquila } & -I \cdot 1 & -1 \\ 1925 & \text { Pictor } & +I \cdot 2 & -25 \\ 1934 & \text { Hercules } & +1 \cdot 4 & +26 \\ 1942 & \text { Puppis } & +0 \cdot 2 & 0\end{array}$

The novae in I901, 1918 and 1942 were fast. The 1942 star was only visible to the unaided eye for about 20 days. After maximum the novae of 1901 and 1918 experienced a decline of about 4 magnitudes in 20 days, followed by irregular fluctuations. The fluctuation in I9OI initially had a range from about +4 to +5.5 . About 50 days after the outburst the minima of the oscillations were fainter than +6 , while the star was brighter than +5.5 for only half of the time. Further observation with the unaided eye would be unlikely. The star of I9r 8 was considerably brighter than that in 1901. About 100 days after the maximum the fluctuations virtually ceased by which time the star had reached +5 . It remained brighter than +5.5 for a further 60 days.

The remaining novae were slow; the brightness remained near maximum for a considerable time. The magnitude of the 1934 star showed much variation but did not fall below +4 for 100 days. After this there was a rapid decline to far below the unaided eye limit. The 1925 nova fell to +3.5 in 20 days, followed by secondary maxima of $+1 \cdot 6$ and $+1 \cdot 8$ some 50 and 60 days after the outburst. It finally fell to +4 after a total of 130 days and to +5 after a further 120 days.

The novae in 1918 and 1925 remained visible to the unaided eye for a period comparable with that recorded for the guest star of $\mathrm{II} 8 \mathrm{I}$. However, the combination of duration of visibility and galactic latitude would prevent any of the stars in Table I, except Nova Aquilae (1918), from being mistaken for a supernova if only brief descriptive details were given, as in early oriental records. Statistics of both modern and ancient novae indicate that stars similar to those in 1918 and 1925 appearing within $5 \mathrm{deg}$. of the galactic equator (the zone in which virtually all known SNR are to be found) must be very rare, occurring perhaps only once in several centuries.

From the material collected by Payne-Gaposchkin (pp. 22-25) on the hundred or so modern galactic novae (mostly very faint) it is evident that fast novae, for which the rate of decline is greater than 0.08 mag. per day, are intrinsically about two magnitudes brighter than slow novae. Further, fast novae are nearly twice as frequent as slow novae. The small sample in Table I contains two stars which 
appeared in a galactic latitude of about 25 deg. Hsi identified 6I probable novae among the early records of guest stars. Many of the observed positions are rather vague and several of his supposed supernovae may well have been novae. However, his results indicate that probably only 15-20 per cent of bright novae appear within $5 \mathrm{deg}$. of the galactic equator.

More than 2000 years of careful observation recorded in the Chinese, Japanese and Korean annals (Hsi; Ho 1962; Hsi \& Po; Chu Sun-il 1968) reveals that, with few exceptions, guest stars were seen either for less than about a month or more than about four months. Stars of short duration were probably novae. Bearing in mind the frequency of supernovae in several external galaxies-during the present century there have been three such explosions in each of the spiral galaxies NGC 3184, 4321, 5236, and 6946 (Zwicky)-we might expect that a number of the guest stars of longer duration were supernovae.

Guest stars observed for a lengthy period are shown in Table II.

TABLe II

Early guest stars observed for several months

\begin{tabular}{|c|c|c|c|}
\hline Year (A.D.) & Constellation & Duration & bII \\
\hline 185 & Centaurus & 8 months & $+3^{\circ}$ \\
\hline 369 & Draconis, etc. & 5 months & \\
\hline 386 & Sagittarius & 4 months & -9 \\
\hline 393 & Scorpius & 7 months & 0 \\
\hline 902 & Cassiopeia & I year & +10 \\
\hline 1006 & Lupus & 10 years (?) & +5 \\
\hline 1054 & Taurus & 2 years & -5 \\
\hline 1181 & Cassiopeia & 6 months & +2 \\
\hline 1572 & Cassiopeia & 18 months & $+\mathbf{I}$ \\
\hline $1592 \mathrm{~A}$ & Cetus & 15 months & -70 \\
\hline $1592 \mathrm{~B}$ & Cassiopeia & 4 months & -3 \\
\hline $1592 \mathrm{C}$ & Cassiopeia & 3 months & -3 \\
\hline 1604 & Ophiuchus & I year & +5 \\
\hline 1664 & Ophiuchus & 8 months & +5 \\
\hline
\end{tabular}

Galactic latitudes in Table II are mainly only approximate but errors are unlikely to exceed $5 \mathrm{deg}$. and may be much less than this. The recorded positions of the 369 star 'at the western wall of Tzü-wei' is too vague to allow even a rough value of $b^{\text {II }}$ to be deduced.

Included in the above list are the well-known supernovae of 1006, 1054, 1572 and 1604. The star 1592 A appeared in a very high galactic latitude and thus must have been a slow nova. It is possible that a nova showing a pronounced secondary maximum may have appeared in Taurus $\left(b^{\mathrm{II}}=-20^{\circ}\right)$ in A.D. 396. In this year a brilliant yellow star 
was seen by the Chinese astronomers for more than 50 days in the summer and reappeared about six months later in the same part of the sky. Taurus was not in conjunction with the Sun during this time. With the exception of the guest star of 369 , all the remaining stars were in a very low galactic latitude. This distribution is quite unlike that of ordinary novae. The only feasible conclusion is that a high proportion of these stars were supernovae.

The explanation of the galactic distribution of guest stars of long observed duration seems to lie in the fundamental difference between the light curves of novae (whether fast or slow) and supernovae. Among the numerous recent extra-galactic supernovae, Zwicky recognizes five types. Type $\mathrm{V}$ is similar to $\eta$ Car (I843). Such stars are best described as irregular variables which reach the absolute magnitude of supernovae at maximum (Minkowski 1968). There has only been one example of type IV. The light curve shown by Zwicky is incomplete. Minkowski regards this and Zwicky's type III (which are also very rare) as extreme examples of type II. All supernova show only a slow rise and fall near the maximum. A supernova of type $I$ is within 3 magnitudes of the maximum for about two months. For supernovae of types II and III this period is about 3-5 months. Those of type $\mathrm{V}$, which are subluminous, hover around the maximum for many months. The entire supernova light curve is smooth. This is much more likely to encourage persistent observation than a curve exhibiting marked irregularities, as is typical of novae.

We may conclude that the evidence is very much in favour of any guest star which was observed for several months being a supernova. If it can be established that the galactic latitude was very low, this virtually rules out the possibility that the star was a slow nova.

In the Azuma Kagami account (g) the guest star was stated to be the only example since the brilliant supernova of A.D. roo6. To what extent comparison should be made is difficult to decide owing to the interval of nearly two centuries between the observations. The 'rays' described are presumably merely the protuberances which appear surrounding all bright stars owing to distortion of light within the eye. That they should be so noticeable suggests that the guest star was considerably brighter than zero magnitude. The silence of all of the remaining accounts on the question of the brightness of the star does not seem to support this conclusion, but it is difficult otherwise to account for the considerable interest aroused by the appearance of the star.

The comparison with Saturn in the Azuma Kagami record is rather unexpected. At the time of discovery in Japan ( $19^{h}$ to $2 I^{h}$ local time) the only planet visible was Mars, fairly high in the south-east. Jupiter 
would become visible near midnight, while Saturn, 17 deg. W of the Sun would only be seen for a short time before sunrise. The magnitude of the planets were about $-I,-2$ and +1 respectively. Perhaps the guest star (which was visible throughout the night) most nearly resembled Saturn in brightness. There is no possibility of the characters representing the various planets being confused. It is most improbable that the reference is to the similarity in colour between the guest star and the planet even though the low altitude of Saturn may have produced colours of atmospheric origin. The description 'bluish-red' needs no qualification, and the observers would have been well aware that the planet is normally white in colour.

The guest star was discovered in Japan one day after the first observation in southern China. Possibly the delay of five days in northern China was the result of unfavourable weather conditions. Kepler's supernova was discovered about eight days before the maximum, during which time the brightness increased by 3 magnitudes (Baade 1943). A rate of increase of this order is characteristic of supernovae of types I-III. Assuming that the star of II8I was about as bright as Saturn at discovery, it is probable that the star became significantly brighter than magnitude o a few days after discovery.

The discrepancy between the periods of observation recorded by the Sung and Chin astronomers is significant. The guest star remained visible in southern China for 24 days longer than in the north. As this was the dry season in the north, the inference is that the brightness remained near the unaided eye limit between the last Chin observation and the last Sung observation. This feature is characteristic of a type I supernova. The schematic type I light curve shown by Zwicky exhibits a uniform decline commencing about 80 days after the maximum and lasting more than 500 days. The time of decline by I magnitude for six type I supernovae varied from 50 to 73 days, i.e. 0.3 to 0.5 mag. in 24 days. Type II light curves show considerable diversity. The few examples of type II supernovae discussed by Zwicky were only observed for up to I30 days owing to their apparent faintness. Three curves show a uniform diminution of light by one magnitude in I2-I 6 days, commencing upwards of 40 days after maximum. Unfortunately, observations on the linear region could only be continued for about 50 days. A further interpolated curve resembles more that of a type I supernova, but, as Minkowski points out, interpolation may well be misleading, and this single example cannot be accepted as proof that the light curve of a supernova of type II can simulate closely that of a supernova of type I. Assuming that the type II linear region is maintained, it appears that the rate of decay of type II is about four times as rapid as that of type I. 
On the more probable assumption that the II8I guest star was of type I, we can attempt a deduction of the magnitude at maximum. At the time of disappearance the star was approaching conjunction with the Sun, but the high declination (about $+60 \mathrm{deg}$. in II8I) would permit two hours of observation at an altitude of more than 30 deg. each morning before nautical twilight commenced. The star is unlikely to have been observed after the magnitude fell below $+5 \cdot 5$; it would then be just another faint star on the verge of visibility (see Section 7). Tycho Brahe, under more favourable circumstances, ceased observation of the 1572 supernova in Cassiopeia when it became fainter than +5.3 (Baade I945). The type I light curves shown by Baade (ibid.) indicate a fall of between 5 and 5.5 mag. in about 180 days. This suggests an apparent magnitude close to zero at maximum, in fair agreement with the other evidence. The magnitude corrected for interstellar absorption may have been considerably brighter than this.

Some remarks on the guest star of I664 seem appropriate; the circumstances surrounding it are rather uncertain. The star is recorded in the Chǔngbo Munhön Pigo (Tsêng Pu Wên Hsien Pei K'ao, a late Korean historical summary) as follows (6/22a):

'In the ninth month of the year chia-ch'en, the fifth year of King Hyŏnjong [19th October-17th November 1664] a guest star was seen above T'ien-chiang. It was as large as Jupiter, yellowish-red in colour and scintillating. It was seen again in the east [i.e. after conjunction with the Sun]. In the fifth month of the following year [13 June-I2 July I665] it was extinguished'.

The star is not recorded in the Hyonjong Sillok (Hsien-tsung Shih $\mathrm{Lu}$ ), the annals of King Hyonjong who reigned at the time, and it does not seem to have been observed elsewhere in the world. The description, position, and time of occurrence agree well with the Korean account of the supernova of 1604 (Kepler's supernova), although this star was not observed in Korea after the third month. It is significant that 1604 and 1664 share the same year name (chia-ch'en) of the oriental sexagenary cycle. There are other instances of error of this sort in the Chǔngbo Munhŏn Pigo (see Section 8). It seems probable that the 1664 record is merely a further account of Kepler's supernova.

\section{OTHER GUEST STARS IN CASSIOPEIA}

The oriental asterisms Wang-liang, Hua-kai and Ch'uan-shê, together with Ko-tao which is not mentioned in the accounts of the guest star, are adjacent to one another in Cassiopeia and cover an area approximately 15 degrees square. Apart from the new star of II81, the 
catalogue of Ho (1962) lists seven guest stars which were seen near these asterisms. The dates of appearance of the stars are: 49 B.c. (seen only in China), 47 B.C. (China), A.D. 722 (Japan), 902 (China), 1005 (China), I572 (China and Korea), and 1592 (Korea). Two guest stars were, in fact, seen in Cassiopeia in 1592 (see below). From A.D. 45I until 1593 eleven comets were sighted in this part of the sky but for each, with the exception of the comet of A.D. I0or, an account of the motion is given. Only the first sighting of the broom star which appeared in A.D. IOOI south of Ch'uan-shê is recorded.

The guest stars of 49 B.C. and A.D. I005 are clearly said to have changed position and thus must have been comets. The star of A.D. 722 was seen by the side of Ko-tao for 5 days before disappearing. This was probably a fast nova. The guest star of 47 B.c. 'stayed at about $5 \mathrm{ft}$ (ch'ih) to the east of Ch'uan-She' for a period of up to a month. This was again probably a fast nova. Its position was much to the east of the area in which the guest star of II8I appeared.

The remaining stars were each seen for several months and are listed in Table II. The new star of 1572, which appeared beside T'sê-hsing (probably $\kappa$ Cas-see Section 6) was Tycho's supernova. This is the radio source ${ }_{3} \mathrm{C}$ 10. The guest star observed in 902 is somewhat enigmatic. A translation of the Hsin T'ang Shu ('New Book of the T'ang Dynasty') record is as follows (32/12a):

'During the first month of the second year of the T'ien-fu reign period [February II to March I2, 902] a guest star like a peach appeared at the Tzŭ-wei beneath [i.e. to the north of] Hua-kai. It gradually moved until it reached Yü-nu. On the day ting-mao [March 2] a meteor started from Wên-ch'ang and reached the guest star; the guest star did not move. On the day chi-szü [March 4] the guest star was at $K^{\prime}$ ang and guarded it. In the next year it still had not disappeared'.

This account is given by Ho (1962). Hsi and Hsi \& Po quote only the description given in the compendium Wên Hsien T'ung K'ao ('Historical Investigation of Public Affairs'), a work by Ma T'uan-lin in 1254. They omit the passage which mentions the motion of the star. $Y u ̈-n u$ (in Draco) is some 30 degrees from Hua-kai, while $K^{\prime}$ ang is immediately to the north of Hua-kai. Whatever the interpretation, the guest star was considerably to the north of the zone within which the star of II8I was seen.

The three guest stars of 1592 (I592A in Cetus and $1592 \mathrm{~B}$ and $1592 \mathrm{C}$ in Cassiopeia) remained fixed. The abridged record in the Chungbo Munhon Pigo (6/2Ib) is rather diffuse and gives the impression that only one guest star was seen in Cassiopeia. The annals of the period (the 
Sonjö Sillok (Hsüan-Tsu Shih Lu) are, however, quite precise. Following the appearance of the star 1592A in Cetus on November 28, the star I592B appeared to the east of Wang-liang on November 30, while $1592 \mathrm{C}$ was sighted to the west of Wang-liang. On almost every day when the weather permitted observation we find the monotonous record: 'During the first watch of the night a guest star was seen within the stars of T'ien-ts'ang [in Cetus], another was seen at the east of Wang-liang and another guest star was seen at the west within the first star'. These stars are in order 1592A, 1592B, I592C. On three occasions $1592 \mathrm{~B}$ was referred to as 'at the east of Wang-liang between the first and second stars' (1593 January 27, February I and February 2). The stars in T'ien-ts'ang and at the west of Wang-liang ( $1592 \mathrm{~A}$ and $\mathrm{I} 592 \mathrm{C}$ ) were not observed after March 4, while the star to the east of Wang-liang was observed once more between the first and second stars on March 29, The star in Cetus was rediscovered later in the year after conjunction with the Sun. We then find a more precise record of position: 'A guest star was seen at the east of T'ien-ts'ang within 3 ts'un of the third star'. According to Wylie (I897) and Hsi \& Po the third star of T'ien-ts'ang is $\theta$ Cet. From below (Section 8) it appears that Io $t s^{\prime}$ un (inches) are to be equated with I $t u$ (degrees of 365.25 to a circle). The guest star was thus probably within 0.3 deg. of $\theta$ Cet (R.A. $\mathrm{I}^{\mathrm{h}} 19^{\mathrm{m}} \mathrm{I}^{\mathrm{s}}$, dec. $-8^{\circ} 42^{\prime}$ at 1900.0).

The three stars of 1592-3 seem to have escaped the vigilance of Tycho Brahe and others in the West, as well as the Chinese and Japanese astronomers, but their authenticity cannot be doubted. The Korean records reveal that cloud and rain interfered considerably with observation of the stars, hence the numerous gaps-often of several days. As both guest stars in Cassiopeia were in very low galactic latitude (about -3 deg.), they are worth investigating as possible supernovae. The period of visibility was, however, only about half of that of the II 8 I star. Further investigation of these stars will be made in Section 10, following a discussion of the asterisms mentioned in the accounts of the II 18 guest star.

From the beginning of the Han Dynasty in China (202 B.C.), records of comets and novae are fairly complete. It is thus unlikely that a supernova visible for several months would pass unnoticed. Most of Cassiopeia has remained within the zone of perpetual apparition for c. $35 \mathrm{deg}$. N. during the past two millenia. The only recorded stars of long duration in this region of sky are those in II8I, 1572 and 1592 (excluding the star in 902 which may have moved, and was much to the north of the other guest stars). We are probably justified in concluding that, apart from 3C 10 (Tycho's supernova of 1572), any SNR in Cassiopeia not associated with the guest stars of 1 I 81 and 1592 must be at least 2000 years old. 


\section{ORIENTAL STAR MAPS}

Early oriental astrography was very detailed but suffered from two major disadvantages. Idealization of form (distortion of an asterism in order to represent better the object named) was exhibited in varying degrees while virtually no attempt was made to differentiate between stars of even widely varying brightness. Star maps constructed after the arrival of the Jesuits (I7th century and later) are much more accurate, but show considerable western influence although retaining the oriental names.

These features make identification of the stars forming the various asterisms a difficult task. The extensive nineteenth century attempts, e.g. by Schlegel (1875) and Wylie, were based on inadequate star catalogues and can only be regarded as partially successful. These often indicate stars fainter than magnitude +6.0 (the usually accepted limit with the unaided eye). In identifying the constellations mentioned in the accounts of the guest star of II8I, I have preferred to make independent deductions with the aid of a modern catalogue (Schlesinger \& Jenkins 1940).

Early oriental planispheres consulted in the course of this work are five in number. These are all of Chinese origin and date from the I3th century A.D. or earlier. Complete or partial copies of four of these maps are shown by Needham (1959, Figs. 99, 100, 104-8). H.G.H.Nelson of the Department of Oriental Printed Books and Manuscripts at the British Museum (private communication) is of the opinion that very little other material from before the Jesuit period is extant in the western world. The Japanese (and also the Koreans) followed Chinese astronomy and astrography, but there appear to be no native Japanese star charts from before the i 7 th century.

The five early star maps (arranged in order of diminishing reliability) are:

(a) A chart of date A.D. I092 contained in Hsin I Hsiang Fa Yao ('New Design for a Mechanised Armillary Sphere and Celestial Globe'), a work by Su Sung. This is probably the earliest printed star chart (Needham, p. 278). The six sections of the chart cover the sky north of about dec. $-57^{\circ}$. There is some evidence of idealization but the overall impression is of a genuine attempt to portray the celestial sphere.

(b) The Soochow chart prepared in A.D. II93 and engraved on stone in 1247 . This shows the entire celestial sphere north of about dec. $-56^{\circ}$ on a zenithal equidistant projection. The edges of the Milky Way (not shown in the Su Sung maps) are represented by irregular lines. This map is rather idealized and several of the asterisms are displaced to allow space for names. An excellent rubbing is shown by Rufus \& Tien (I945). 
(c) A Korean planisphere of A.D. 1395. This is an exact copy of a very early Chinese star map. The original stone engraving was presented to the King of Koguryó (one of the three kingdoms of early Korea) by the Emperor of China. The stone was lost in a river when Koguryo fell in A.D. 672. However, a new engraving based on a rubbing of the stone was made in 1395 . This and a later copy (I668) still survive. The position of the pole suggests an epoch c. I00 B.C. (Rufus 1913).

Knobel (1909) describes a planisphere (possibly as late as the I9th century) which was found in a Japanese junk. Rufus \& Chao (1944) draw attention to the fact that this is either a careful copy of the Korean map or shared a common origin with it. Idealization of certain constellations is very pronounced, but evidently the original Chinese astrography was still a valuable aid to navigation about 2000 years later.

Both maps represent the entire celestial sphere north of dec. $-55^{\circ}$ on a zenithal equidistant projection and show clearly the Milky Way. No names are inscribed on the Japanese version. The illustrations given by Knobel are a valuable supplement to those of the Korean map given by Rufus (1915) and Rufus \& Chao.

(d) The Tunhuang chart (A.D. 940). The star maps which form part of the collection of manuscripts from Tunhuang (Brit. Mus. Stein No. 3326) are probably the earliest extant in the world (Needham, p. 270). There are 13 sections representing the portion of the celestial sphere visible in China. The Milky Way is not shown. Although very sketchy, the maps are valuable as an independent reference.

(e) Hsing Ching ('Star Manual'). This is probably a compilation of pre-Han and Han catalogues with later additions up to about A.D. 600. It forms part of the Taoist Canon. Each asterism is shown in a separate diagram so that the work is virtually useless for the present purpose.

A useful supplement to the above collection is an accurate planisphere prepared by Jesuit astronomers in I745. This is reproduced by Rigge (I9I5). It consists of two separate maps showing the entire northern and southern hemispheres. In most cases identification can be established by actual measurement of position. However, the chart represents Chinese astrography, somewhat westernized, as it was more than 500 years after the appearance of the guest star.

In addition to the star maps, I have made use of the detailed descriptions of the constellations given in chapter II of the Chin Shu ('Book of the Chin (Tsin) Dynasty'). The astronomical chapters of this work were written about A.D. 635 by Li Shun-fêng, an eminent astronomer and mathematician. They have been translated, with commentary, by Ho (I966). 


\section{THE LUNAR MANSION K'UEI}

With the exception of the Tunhuang and Hsing Ching charts, all of the star maps consulted show the celestial sphere divided into 28 unequal zones, each defining a range of right ascensions. These lunar mansions (hsiu) take the names of a series of 28 asterisms lying near the celestial equator. The western boundary of each zone is the meridian of a selected star of the same name. Reference to a particular $h s i u$ was common practice in the far east when describing the location of a comet, nova, eclipse, etc. The reference to $K^{\prime} u e i$ hsiu, which occurs only in the Sung Shih record of the guest star of I $18 \mathrm{r}$, is thus intended to give an indication of the right ascension of the star. The $h s i u$ were numbered eastward from Chüeh ( $\alpha$ and $\beta$ Vir). $K^{\prime}$ 'uei is the 15th lunar mansion.

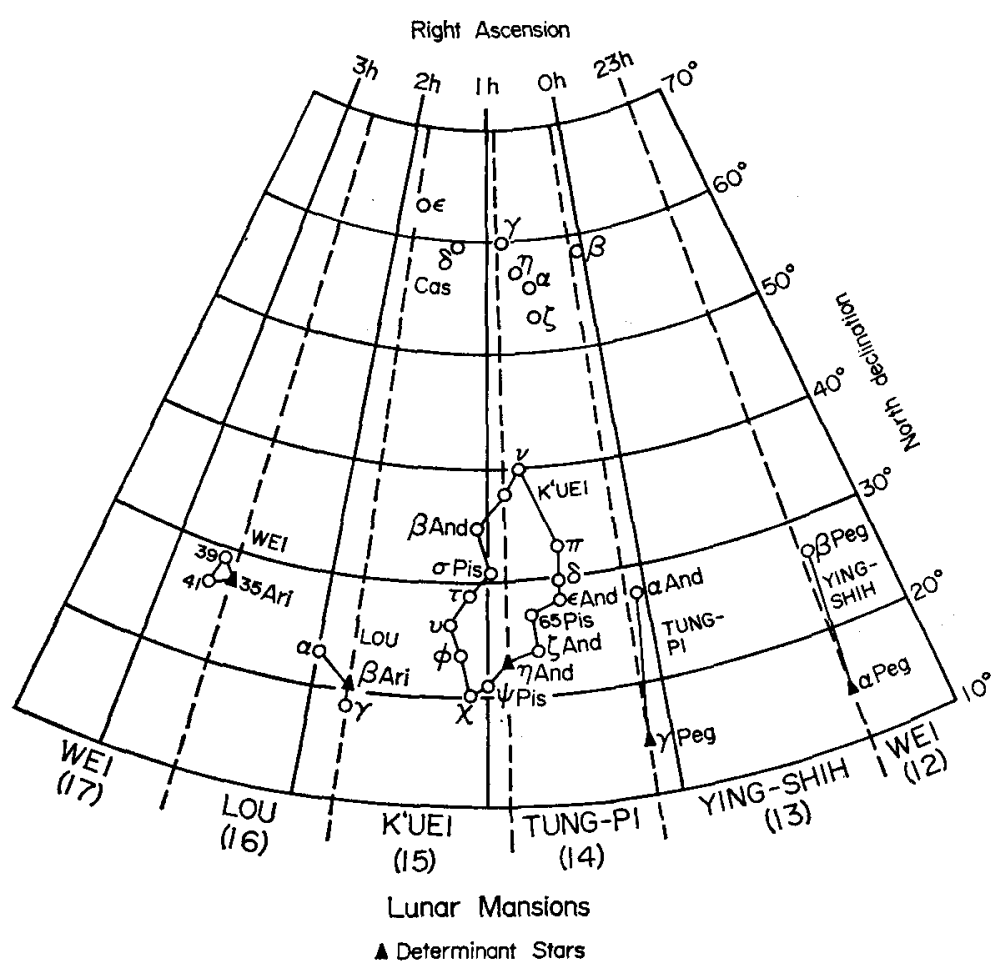

Fro. I. Stars of the $13^{\text {th }}$ to 17 th lunar mansions in relation to the principal stars of Cassiopeia.

Fig. I shows the stars forming the lunar mansions for the region of the sky from $22^{\mathrm{h}} 30^{\mathrm{m}}$ to $3^{\mathrm{h}} 30^{\mathrm{m}}$ right ascension at $1900 \cdot 0$. The Su Sung, Soochow, Korean and Jesuit maps are agreed about the constituent 
stars of the asterisms Ying-shih, Tung-pi, Lou and Wei. However, there is minor disagreement over $K^{\prime} u e i$ (which contains 16 stars according to the Chin Shu description). I have chosen the most probable formation. The principal stars of Cassiopeia, in which the guest star was seen, are marked in Fig. I. The hsiu boundaries are denoted by broken lines. The Soochow and Korean charts disagree with the Su Sung and Jesuit identification of $a$ Peg as the determinant star of Ying-shih, seemingly indicating $\beta$ Peg instead. The Korean chart alone favours $\zeta$ And (rather than $\eta$ And) as the determinant star of K'uei. Otherwise there is no discord. In Fig. I, I have adopted a Peg and $\eta$ And as defining these two hsiu.

A star defining a lunar mansion is by no means necessarily the brightest in a group. The determinant stars were chosen by the early Chinese because of their correlation with circumpolar phenomena (Needham, p. 239). Owing to this method of selection, a considerable part of a constellation may lie to the west of the hsiu of the same name. This is especially true of $K^{\prime} u e i$. The position of the guest star within the mansion is not recorded. In view of the northerly aspect of the asterism $K^{\prime} u e i$ it seems possible that if the star appeared in Tung-pi it might be mistakenly assigned to $K^{\prime} u e i$. There would be plenty of time in which to correct an error of this sort, but it must not be assumed that the boundaries of the lunar mansions are absolute.

\section{ASTERISMS MENTIONED IN THE GUEST STAR RECORDS}

Fig. 2 is a detailed chart of the Cassiopeia region for the epoch 1900.0. The positions of all stars brighter than visual magnitude +6.0 in the zone R.A. $22^{\mathrm{h}} 30^{\mathrm{m}}$ to $30^{\mathrm{h}} 30^{\mathrm{m}}$, Dec. $+55^{\circ}$ to $+75^{\circ}$ are shown. Numbers represent magnitudes-for the few variable stars the range is given. Bayer stars are denoted by the appropriate Greek letter. Only a few stars have annual proper motions in excess of about $O$ " 1 . These are $\beta$ Cas, $\eta$ Cas, $\delta$ Cas and two stars of magnitude $+5.7-$ BD $+56^{\circ} 2966$ (R.A. $23^{\mathrm{h}} 8^{\mathrm{m}}$, Dec. $\left.+57^{\circ}\right)$ and BD $+63^{\circ} 23^{\circ}\left(\mathrm{I}^{\mathrm{h}} 40^{\mathrm{m}}\right.$, $\left.+63^{\circ}\right)$. The proper motions are in order: $0^{\prime \prime} \cdot 5,1^{\prime \prime} \cdot 2,0^{\prime \prime} \cdot 3,2^{\prime \prime} \cdot 1$ and $0^{\prime \prime} \cdot 6$. The aspect of this part of the sky has thus remained virtually unchanged since the appearance of the guest star. The hsiu boundaries at I I8I, corrected for precession to $1900 \cdot 0$, are indicated by broken lines on Fig. 2. The presence of these boundaries on the four most reliable charts is of considerable importance in determining the stars forming the asterisms which are mentioned in the accounts of the guest star.

The references to Ch'uan-she, Hua-kai and Wang-liang in the Chinese and Japanese accounts of the guest star are quite independent. All of the star maps consulted show these constellations, but the form of each differs to a greater or lesser degree from one map to another. 
The stars forming Hua-kai and Wang-liang can be identified with confidence, for the shape of both of these asterisms is very well defined. However Ch'uan-shê is very extensive and consists of faint stars. In consequence, identification of the constituent stars is more difficult. There are two references to this asterism in the guest star accountsfrom southern China (Sung Shih) and Japan (Dainihonshi or Meigetsuki). It is fortunate that the Chinese observation is the more valuable (see Section 8), for three of the star charts used in this investigation were constructed in China at about this time.

Anticipating the outcome of this investigation, it is apparent that stars brighter than mag. $+5^{\circ} 0$ were seldom omitted from the oriental asterisms. Both Hua-kai and Ch'uan-shê certainly contain several stars as faint as +5.5 but none fainter than this. As already mentioned, Hua-kai can be reconstructed with confidence. Two of the stars of this group are of magnitude $5 \cdot 5$, while $Y \ddot{u}-k u e i$, the 23 rd lunar mansion (the trapezium in Cancer surrounding Praesepe), contains two stars of magnitude 5.5 and 5.6 . The limit for a recognizable feature seems to lie very close to magnitude 5.5 ; stars fainter than this can probably be only glimpsed in favourable conditions. The Chinese astronomers, like their western counterparts, counted only seven stars in the Pleiades (Mao, the I8th lunar mansion). These must have been 17 Tau (mag. + 3.8), I9 (4.4), $20(4 \cdot 0), 23(4 \cdot 3), 25 \eta(3.0), 27(3.8)$ and either 28 Tau $(5 \cdot 2)$ or 16 Tau $(5 \cdot 4)$. Other stars in this cluster brighter than $+6 \cdot 0$ are I 8 Tau $(5 \cdot 6)$, 2I Tau (5.9) and BD $+22^{\circ} 5^{6} 3(5.5)$. All of these stars must have been overlooked.

As well as the constellations Ch'uan-shê, Hua-kai and Wang-liang it is necessary to attempt reconstruction of Ko-tao which lies close to all of these formations. On the Su Sung star chart, Ko-tao actually intersects Ch'uan-shê, a very rare occurrence. Two isolated stars, Tsê-hsing which forms part of Wang-liang, and $\mathrm{Fu}$-lu, part of Ko-tao, are also included in this investigation.

(a) Hua-kai

According to the Chin Shu (Ho 1966, p. 69), 'On top of (the star) Ta-ti [Polaris] and suspended above the Imperial Throne are the seven stars of Hua-kai ('Gilded Canopy'). Below [i.e. to the north of] Hua-kai and forming its handle are the nine stars of $K$ 'ang ('Flagstaff')'. The shape of this compact group is so characteristic that although the constituent stars are rather faint, it is immediately recognizable on all of the star maps consulted. The formation is most carefully portrayed on the Jesuit, Su Sung and Soochow maps. The Jesuit map shows Hua-kai lying in K'uei hsiu. On both the Soochow and Su Sung maps the asterism is situated in Lou, the adjacent lunar mansion, but this is obviously an error. The stars are without doubt 32 Cas (mag. 5*3), 
$36 \psi\left(5^{\circ} 0\right), 40(5 \cdot 5), 42(5 \cdot 3), 4 \cdot 3(5 \cdot 5), 46 \omega\left(5^{\circ} 0\right), 48(4 \cdot 6)$ and 50 Cas (4. I). The star 40 Cas, at the north, probably forms part of the 'flagstaff' ( $\left.K^{\prime} a n g\right)$, which extends much to the north.

\section{(b) Wang-liang and T'sê-hsing}

Named after a famous early charioteer, this group represents a chariot and a team of four horses, together with a whip. By defin ition, 'Wang-liang, comprising five stars north of $K$ 'uei (I 5 th lunar mansion) lies at the centre of the Milky Way [i.e. at its northernmost part] and (denotes) the charioteer of the Son of Heaven. Four of these stars are known as T'ien-szú ('Celestial Quadriga') while the one by the side is called Wang-liang. . . . The single star called T'sê-hsing ('Whip Star'), the whip used by the Wang-liang . . . is found by the side of Wang-liang' (op. cit, p. 89). On all six maps $\beta$ Cas represents Wangliang himself. The four main charts-(a) to (c) and the Jesuit chartseem agreed that the remaining stars (representing the team and whip) are (in order of R.A.), $\kappa, \alpha, \eta, v$ (regarding $v^{1}$ and $v^{2}$ as one star) and $\gamma$ Cas. Whereas on the Jesuit planisphere $\gamma$ Cas is named as $T$ 'sê-hsing, the other three charts point to $\kappa$ Cas at the north. Support for $\kappa$ Cas as the whip comes from records of the supernova of 1572 . Both Chinese and Korean records describe this star as beside T'sê-hsing. The corresponding radio source $\left({ }_{3} \mathrm{C} \mathrm{IO}\right)$ is only $\mathrm{I} \cdot 2 \mathrm{deg}$. north-west of $\kappa$ Cas. The distance from $\gamma$ Cas is $5 \mathrm{deg}$. I have adopted $\kappa$ Cas as the whip.

\section{c) Ko-tao and Fu-lu}

These asterisms are described in the Chin-Shu as follows. 'Ko-tao ('Hanging Gallery'), consisting of six stars in front of Wang-liang, like a flying path (across mountains), stretches from the 'Purple Palace' (the circle of perpetual visibility) to the Milky Way. ... The star Fu-lu ('Auxiliary Road') south of Ko-tao (represents) the alternative side-routes' (ibid).

Both Ko-tao and Fu-lu show considerable diversity of form on the various charts. On the Jesuit map the stars of Ko-tao are certainly $\iota, \epsilon, \chi, \phi, \theta$ and either $\xi$ or $\nu$ Cas. $F u$ - $l u$ is $\zeta$ Cas. These last three stars lie to the south of the region covered by Fig. 2. It is clear from the relative positions of $،$ Cas, $\epsilon$ Cas and BD $+63^{\circ} 265$ (the 5.2 mag. star lying between $\iota$ and $\epsilon$ Cas) that BD $+63^{\circ} 265$ forms part of Ch'uan-shê. On the Su Sung map the five stars of Ko-tao are ı, $\epsilon, \chi, \phi$ and $\theta$ Cas, while $F u$-lu is $\delta$ Cas. Here Ch'uan-shê intersects $K o$-tao between ' and $\epsilon$ Cas. Ch'uan-she is very idealized, but it is clear from the position of the $K^{\prime}$ uei-Lou boundary that BD $+63^{\circ} 265$ is one of the 
stars of this asterism. The Korean planisphere appears to confirm the Jesuit identification of $\mathrm{Ko}$-tao but replaces $\zeta$ Cass by $\chi$ Cass as Fu-lu.

The remaining representations are very idealized. The Soochow chart points to $\delta$ Cas as $F u$-lu but Ko-tao is very distorted, most probably containing $\theta, \phi, \chi$ and $\epsilon$ Cas. On the Tunhuang map $\mathrm{Ko}$-tao and $\mathrm{Fu}$-lu form a single asterism, presumably, from its shape, consisting of $\iota, \epsilon, \chi, \delta, \phi$ and $\theta$ Cas.

The formation shown in Fig. 2 seems to be the most probable one, but obviously there is considerable doubt about $\mathrm{Fu}$-lu. However, we can be fairly confident that within the boundary of Fig. 2 all of the stars $\iota, \epsilon, \chi, \delta, \phi$ and $\theta$ Cas, and only these, are members of the two asterisms.

\section{(d) Ch'uan-shê}

'Ch'uan-shê ('Inns'), comprising nine stars and situated above [i.e. to the south of] Hua-kai near the Milky Way, represents the guesthouses...' (op cit. p. 69). This undistinguished constellation is very extensive. All of the maps (except those in the Hsing Ching) show Ch'uan-shê situated immediately to the south of Hua-kai. On the Jesuit, Soochow and Korean maps the asterism is represented as lying to the north of, and entirely outside, the Milky Way-in accord with the Chin Shu description.

The Jesuit representation of Ch'uan-shê shows only five stars. Measurement of position indicates that four of the stars are ro Cas (mag. 5.5), $\mathrm{BD}+63^{\circ} 99(5 \cdot 5), \mathrm{BD}+63^{\circ} \mathrm{I} 49(5 \cdot 5)$ and $\mathrm{BD}+63^{\circ} 265(5 \cdot 2)$. The fifth is probably 32 Cas $(5.5)$ displaced so that it is in line with the others. This formation is shown in Fig. 2. On both the Soochow and Su Sung maps Ch'uan-shê ranges much farther to the east, but the five westernmost stars appear to be identical with those on the Jesuit planisphere. The gap between BD $63^{\circ} 265$ and the first of the eastern stars, probably BD $65^{\circ} 340$ (mag. 4.8 ), is clearly shown in each case. On the Soochow chart the group has a zigzag pattern, seemingly an attempt to portray the true configuration.

All of these stars are faint but it is difficult to accept alternative identifications; there are no other suitable stars between Hua-kai and the Milky Way. The star $\kappa$ Cass, identified above as $T$ 'sê-hsing is certainly not part of Ch'uan-shê for it lies well within the northern edge of the Milky Way. The occurrence of two independent references to Ch'uan-she in the accounts of the guest star suggests that it was a well-known formation. This is apparent from the frequent allusions to the constellation in cometary records (e.g. in A.D. 45I, 626, I337 and I593). 
The expression fan ('invade', 'offend', 'trespass against', etc.) which occurs in the Sung Shih account of the guest star is very frequently used in far eastern records to describe conjunctions of the Moon and planets with one another and with stars. The term is defined in chapter 157 of the Wu Pei Chih ('Treatise on Armament Technology'dated I628) in several ways. These are mainly descriptive, but a numerical definition is as follows: 'When (a celestial body) comes within 7 ts' $u$ m of (another) such that their rays extend towards each other' (Ho, 1966, p. 37). The Chin Shu underlines the astrological significance of the expression: 'When the planets are within a distance of seven inches ( $t s^{\prime}$ un) from each other the omen will assuredly be fulfilled' (op. cit., p. 127). This apparent confusion of linear and angular units is clarified by the $\mathrm{Wu} \mathrm{Pei}$ Chih in its discussion of planetary motions (op. cit. p. 35). Fast motion (su hsing) is defined as 'movement of 5 ts'un to I ch'ih (per day)', while haste (chi) is 'Movement of I $t u$ (degree) per day'. Slow planetary motion ( $c h$ 'ih hsing) is defined as 'movement of $I$ or 2 ts'un a day'. Comparison of these rates with the mean daily motions of the planets suggests that ch'ih (of ro ts'un) and $t u$ (degrees, numbering $365 \cdot 25$ to a circle) are synonymous.

An investigation of numerous Chinese and Korean observations of the Moon and planets invading one another, or stars, reveals that fan was usually used when the least separation was about half a degree, and only very rarely for a minimum approach of more than a degree (Stephenson, in preparation). This seems to confirm the figure of 0.7 deg. deduced from the $W u P e i$ Chih. When an occultation occurred the more precise terms yen (conceal), shih (eclipse), or $j u$ (enter) were used. Occasionally very close approaches of planets to one another, or to stars, were described in these ways. An unexpected outcome of the investigation is the evidence that although the civil day began at midnight, the astronomers of both countries adopted an astronomical day beginning at sunrise-normally a readily defined moment.

The use of the expression fan does not necessarily imply motion. In the context of the guest star record the reference is to a star, not previously seen, suddenly appearing close to one of the permanent members of a constellation. In order to examine the reliability of the term as it applied to Sung observations, I have analysed a series of planetary conjunctions which were observed between 1167 and 1200 . These are recorded in the Sung Shih (56/4a) and cover four reign periods. In each case it is stated that one planet invaded another. The results of this analysis are shown in Table III. 
TABLE III

Planetary conjunctions recorded in the Sung Shih

\begin{tabular}{|c|c|c|c|c|c|c|}
\hline $\begin{array}{l}\text { Ref. } \\
\text { No. }\end{array}$ & $\begin{array}{l}\text { Recorded } \\
\text { date }\end{array}$ & Planets & $\begin{array}{c}\text { Actual } \\
\text { date }\end{array}$ & Time & Distance & Elong. \\
\hline I & I167 Dec. 24 & $\mathrm{~V}, \mathrm{~J}$ & Dec. 24 & $20^{h}$ & $0^{\circ} .57$ & $53^{\circ} \mathrm{E}$ \\
\hline 2 & I 68 Apr. I4 & $\mathbf{M}, \mathbf{S}$ & Apr. I8 & 2 & 0.65 & $78 \mathrm{~W}$ \\
\hline 3 & I I70 Sep. 9 & $\mathbf{M}, \mathrm{J}$ & Sep. I3 & 5 & 0.09 & $106 \mathrm{~W}$ \\
\hline 4 & I 172 Jun. 18 & $\mathbf{V}, \mathbf{J}$ & Jun. 18 & 20 & 0.97 & $17 \mathrm{~W}$ \\
\hline 5 & I I73 May I I & $\mathbf{M}, \mathbf{J}$ & May 12 & 21 & 0.61 & $71 \mathrm{E}$ \\
\hline 6 & I I73 Sep. 5 & $\mathrm{~V}, \mathrm{~J}$ & Sep. 6 & 5 & 0.32 & $18 \mathrm{~W}$ \\
\hline 7 & I175 Oct. 25 & V, M & Oct. 25 & 18 & $0.4 \mathrm{I}$ & $32 \mathrm{E}$ \\
\hline 8 & I18I Aug. I4 & $V, S$ & Aug. I5 & 4 & 0.16 & $23 \mathrm{~W}$ \\
\hline 9 & I I84 Aug. 3I & $\mathrm{V}, \mathrm{J}$ & Sep. I & 4 & 0.69 & $35 \mathrm{~W}$ \\
\hline 10 & I1 87 Nov. 14 & $\mathrm{~V}, \mathrm{~S}$ & Nov. 15 & 6 & 0.47 & $36 \mathrm{~W}$ \\
\hline II & II89 May 22 & V, M & Jun. $\quad 2$ & 4 & $I \cdot 52$ & $40 \mathrm{~W}$ \\
\hline I 2 & II9I Dec. 3I & $\mathbf{V}, \mathbf{J}$ & Dec. 31 & 18 & 0.38 & $45 \mathrm{E}$ \\
\hline 13 & I 95 Oct. I I & $\mathrm{V}, \mathrm{M}$ & Oct. I 2 & 5 & 0.30 & $4 \mathrm{I} \mathrm{W}$ \\
\hline 14 & 1198 Nov. 18 & $\mathrm{~V}, \mathrm{~J}$ & Nov. 19 & 6 & 1.06 & $46 \mathrm{~W}$ \\
\hline 15 & I I99 Dec. 2 & $\mathbf{M}, \mathbf{J}$ & Dec. 5 & 6 & 0.56 & $34 \mathrm{~W}$ \\
\hline I6 & I 200 Jan. & V, S & Jan. I & 18 & 0.95 & \\
\hline 17 & I 200 May 22 & $\mathbf{M}, \mathbf{S}$ & May 25 & 4 & 0.26 & $83 \mathrm{~W}$ \\
\hline
\end{tabular}

Column 2 of Table III gives the recorded date converted to the Julian Calendar using the tables of Hsüeh Chung-san \& Ou-yang (1956).

Column 3 gives the initial letter of one of the four planets Venus, Mars, Jupiter and Saturn (observations of Mercury are extremely rare).

Columns 4 and 5 give the calculated date and local time (to the nearest hour) of closest visible approach. The time chosen is the most favourable time for observation of the conjunction, and is within 12 hours of the time of closest approach. Positions of Venus and Mars were computed using the orbital elements given by Newcomb (I895). For each planet an unperturbed orbit was assumed. This treatment is quite adequate for the present purpose. Positions of the slower moving outer planets were found by interpolation in Tuckerman's Tables (1964).

Column 6 gives the separation of the two planets at the time given in column 5. Column 7 gives the elongation of the planets east or west of the Sun.

Of the 17 observations, the recorded dates of II are exact (I, 4, 6, 7, $8,9,10,12,13,14$ and I6). For observation No. II the date is Io days in error. This implies that the cyclical day $i$-wei of the record should be replaced by $i$-szŭ although the characters wei and $s z \breve{u}$ are quite dissimilar. This observation appears to be very unreliable, for the closest visible approach $(1.52 \mathrm{deg}$.) is about $0.5 \mathrm{deg}$. larger than any other in Table III. For the remaining 5 observations $(2,3,4$, I5 and 17) the Sung-Shih records a date differing by up to 3 days 
from the calculated date. If the record gives the true date of observation, the amended separations (in degrees) are respectively $\mathrm{I} \cdot 3 \mathrm{I}, 0.88$, $0 \cdot 78, \mathrm{I} \cdot 06$ and $\mathrm{I} \cdot 35$. It seems quite possible that cloud interfered with some of the observations. However, the average of these 5 results is about 2.5 times that of the corresponding figures in Table III and twice that of the remaining quantities in the table (excluding No. II). An error in the recorded date is thus a more probably assumption. This is a common occurrence in lunar records, for which the intended date can be established with certainty owing to the rapid motion of the Moon. The Sung planetary observations thus indicate a maximum separation of about a degree for the term fan to be applicable, in accord with the limit deduced from other oriental observations.

An interesting Korean observation of the planet Venus invading the guest star of 1604-5 (Kepler's supernova) is recorded in the Sonjo Sillok on a date which corresponds to I605 January 20 (Gregorian). This report is copied in the Chüngbo Munhorn Pigo (6/2Ib) but the year is mistakenly given as the 33rd year of Sonjo (I600-I) rather than the 37 th, as in the Sonjo Sillok. The catalogues of Ho (1962) and Hsi $\&$ Po use the Chüngbo Munhon Pigo account and thus appear to describe a separate guest star in 1600-I. Accurate measurements of the position of the supernova by Kepler and Fabricius give the coordinates at $1605^{\circ} \circ$ as R.A. $17^{\text {h }} 7^{\text {m }} 3,^{\text {s }}$ dec. $-21^{\circ} 5^{\prime} \cdot 3$, with a mean error of about $\pm I^{\prime}$ (Baade I943). Calculation shows that before dawn on January 21 (astronomical date January 20) in 1605 , Venus and the star would be seen only $0.5 \mathrm{I} \mathrm{deg}$. apart. It thus seems probable that throughout the far east a sighting tube was used in order to decide when the expression fan was appropriate.

The guest star of II8I invaded the stars of Ch'uan-shê. Although there are no Sung records of planets invading constellations (rather than single specified stars), an investigation of similar records in the Chin Shu shows that in each case there was an approach of the planet to within a degree of any one of the stars of the specified constellation occurred.

\section{DISCRETE RADIO SOURCES IN FIG. 2}

The radio sources listed in the revised ${ }_{3} \mathrm{C}$ catalogue at $178 \mathrm{MHz}$ (Bennett, 1962) which lay within the region R.A. $22^{\mathrm{h}} 30^{\mathrm{m}}$ to $3^{\mathrm{h}} 30^{\mathrm{m}}$, Dec. $+55^{\circ}$ to $+75^{\circ}$ at $1900 \cdot 0$ are represented by squares in Fig. 2. The more accurate $4 \mathrm{C}$ positions of these sources are used. The source ${ }_{3} \mathrm{C}$ I4. I was separated into two widely-spaced components in the ${ }_{4} \mathrm{C}$ survey $\left(4 \mathrm{C} 58.02\right.$ and $\left.{ }_{4} \mathrm{C} 60^{\circ} 02\right)$. The radio sources shown in Fig. 2 include all of those in $4 \mathrm{C}$ of flux density greater than about io f.u. (I f.u. $=\mathrm{IO}^{-26} \mathrm{~W} \mathrm{~m}^{-2} \mathrm{~Hz}^{-2}$ ) at $178 \mathrm{MHz}$ (compared with the threshold 
value of $2 \cdot 0$ f.u.). The section of the galactic equator $\left(b_{1}^{\pi}=0^{\circ}\right)$ in the range of galactic longitudes to $1^{\mathrm{II}}=105^{\circ}$ to $\mathrm{I}^{\mathrm{II}}=145^{\circ}$ is shown.

The $4 \mathrm{C}$ and revised $3 \mathrm{C}$ surveys (like their earlier counterparts) were interferometric. Sources of apparent diameter more than about $\mathrm{I}^{\prime} \cdot 5$ arc were observed with reduced intensity. The supernovae of 1054 and I572 are of comparable age to the II8I outburst. The remnants of these explosions ( $3 \mathrm{C}_{10}$ and ${ }_{3} \mathrm{C}_{144}$ ) are of diameter about $5^{\prime}$ arc. These were observed with considerably diminished intensity in the surveys. SNR of angular extent about I deg., such as CTA I and

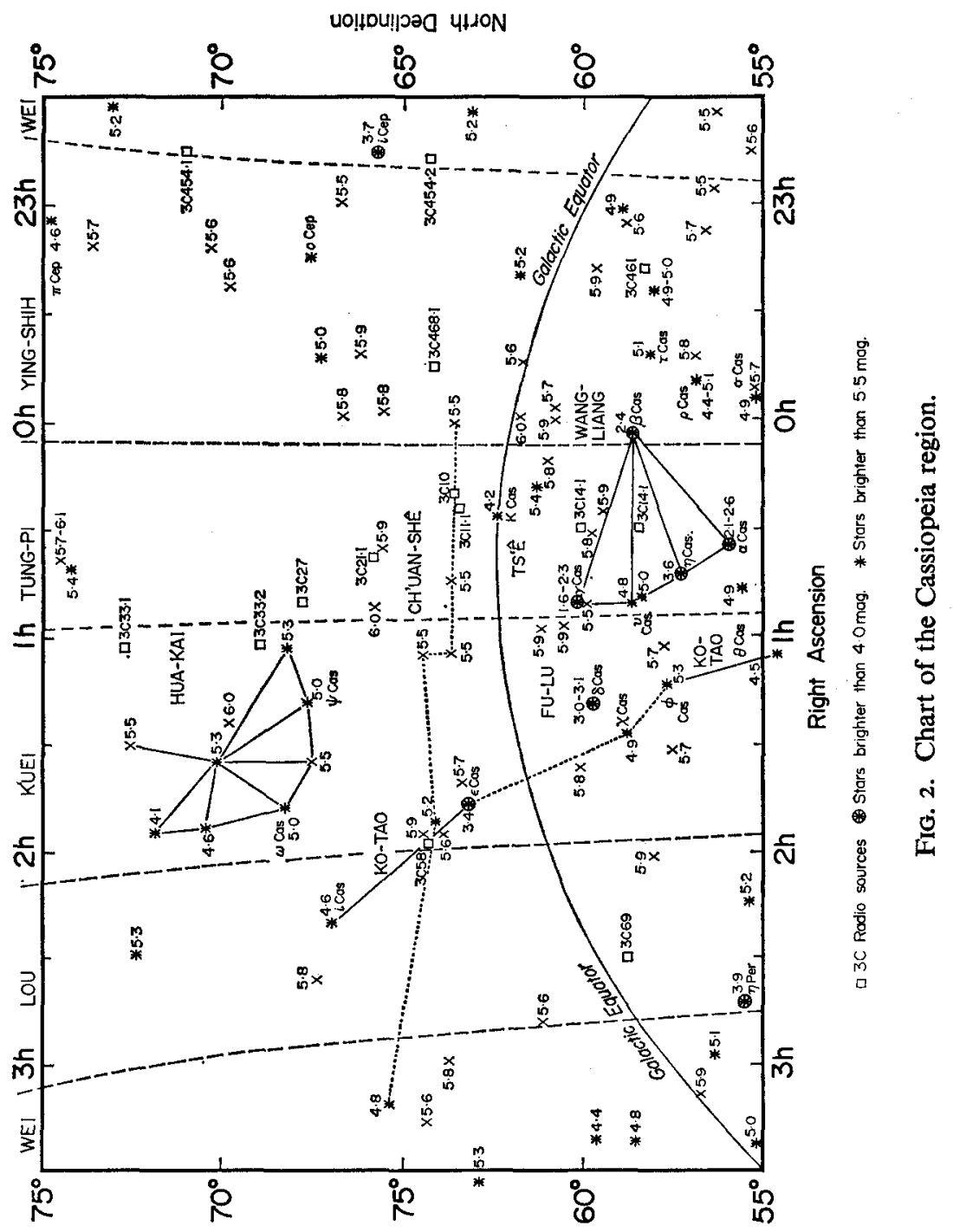


$\mathrm{HB} 3$, were not detected even in the $4 \mathrm{C}$ survey (Caswell 1967). Outbursts responsible for such extended sources, which are not of high integrated flux density, would appear to be very ancient and thus cannot be associated with the guest star of II8I. I have not included these SNR in Fig. 2. The majority of extragalactic sources have apparent diameters of no more than a few seconds of arc. Sources of this nature which were detected in the Cambridge interferometric surveys were observed with undiminished intensity.

Using an average of about one ${ }_{4} \mathrm{C}$ extra-galactic source in any 5 square degrees of sky, Caswell (1969) showed that the $4 \mathrm{C}$ survey was very incomplete in the vicinity of Cassiopeia $\mathrm{A}\left({ }_{3} \mathrm{C} 46 \mathrm{I}\right)$. No source is catalogued within $5 \mathrm{deg}$. of this extremely powerful emitter. However, a count for the area in Fig. 2 east of $0^{h}$ R.A. shows that coverage for this region is virtually complete.

Within the zone between $61^{\circ} \cdot 5$ and $67^{\circ} \cdot 5$ Dec. and $o^{\mathrm{h}}$ to $3^{\mathrm{h}} 30^{\mathrm{m}}$ R.A. there is no $4 \mathrm{C}$ source more intense than $3.5 \mathrm{f}$.u. (less than twice the threshold value of the survey) other than those indicated in Fig. 2. For this region, inside which the 1900.0 position of the guest star must be found, it is unlikely that any discrete galactic source is overlooked.

The apparent stellar magnitudes of the supernovae of 1572 and 1604 at maximum are fairly well known. These lie close to -4 and -2 (Baade 1944), compared with o for the guest star of II8I. The total flux densities at $178 \mathrm{MHz}$ of the two SNR ${ }_{3} \mathrm{C}$ 10 (Tycho) and ${ }_{3} \mathrm{C} 358$ (Kepler) are about 145 and 50 f.u. This suggests a total flux density of roughly Io f.u. for the remnant of the I I 8 I guest star at this frequency, but of course no allowance is made for interstellar absorption of light or varying spectral index.

\section{IO. IDENTIFICATION OF A RADIO SOURCE \\ ASSOCIATED WITH THE GUEST STAR}

From their low galactic latitudes and lengthy period of observation

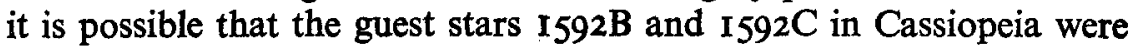
supernovae. The records are, however, too brief to allow any attempt at classification. If both stars were supernovae of type I the apparent magnitude at maximum, deduced from the recorded period visibility (I 9 and 90 days), would be about +1.5 for $1592 \mathrm{~B}$ and +2.0 for 1592C. The form of the type II light curve is very variable, but if the stars were of this type they can scarcely have been any brighter than $+\mathrm{r} \cdot 5$ and may well have been fainter than this, for they escaped detection in the West as well as in China and Japan.

The first star of Wang-liang is undoubtedly $\beta$ Cas. This represents Wang-liang himself. The star 1592B was observed to the east of Wang-liang between the first and second stars. Which of the remaining 
four stars of the asterism is the second star is uncertain. In spite of this, the position of both components of ${ }_{3} \mathrm{C} \mathrm{I4}_{14} \mathrm{I}\left({ }_{4} \mathrm{C} 58.02\right)$ and $(4 \mathrm{C} 60.02)$ would fit the description well. That both radio sources were observed with undiminished intensities (respectively I6.I and 13.2 f.u. at $178 \mathrm{MHz}$ ) in the ${ }_{4} \mathrm{C}$ survey indicates that the apparent diameters are less than $+I^{\prime} \cdot 5$. However, such a recent and faint outburst as I592B might well produce a radio source answering this description. Very little is known about $4 \mathrm{C} 60.02$, but the spectral index a (defined in the usual way, i.e. $\left.\mathrm{I}\left(\nu^{\alpha}\right) \sim v\right)$ of $4 \mathrm{C} 58.02$ is negative, characteristic of a non-thermal source such as a SNR. Kellermann et al. (1969) gives the value of $a$ over the ranges $178-750 \mathrm{MHz}$ and $750 \mathrm{MHz}-5 \mathrm{GHz}$ for this source as $a(178 / 750)=-0.78 \pm 0.17$ and $\alpha(750 / 5000)=$ $-0.99 \pm 0.04$. The proximity to the galactic equator of both sources $\left(b^{\mathrm{II}}=-4^{\circ}\right.$ and $\left.-2^{\circ}\right)$ makes them well worth investigating as possible SNR.

On the assumption of high interstellar absorption of light, Chu Sun-il has attempted to identify the guest star $1592 \mathrm{C}$ with the very powerful radio source Cas $A\left({ }_{3} C_{46}\right)$. This source seems rather far from $\beta$ Cas (about $4 \mathrm{deg}$.) to be described as 'within the first star'. The time of outburst of the supernova associated with Cas $\mathrm{A}$ has been deduced, from the rate of expansion of the remnant, as no earlier than A.D. $1702 \pm 14$ (Minkowski). However, 1592C is the only known temporary star of about the right age and location. Obviously a decision cannot be made.

The guest star of II 81 was visible for a much longer time than $1592 \mathrm{~B}$ and $1592 \mathrm{C}$ and attracted considerable attention. We can thus be very confident of the existence of a SNR associated with this star. Taking the various oriental descriptions of the position of the guest star in a general sense-'in $K^{\prime}$ uei hsiu and invading the stars of Ch'uan-she' (Sung Shih), 'at Hua-kai' (Chin Shih), 'near Wang-liang and guarding Ch'uan-shê' (Dainihonshi and Meigetsuki)-it is evident from Fig. 2 that only three radio sources can be considered for further investigation. These are ${ }_{3} \mathrm{C}$ II I I, $3 \mathrm{C} 2 \mathrm{I} \cdot \mathrm{I}$ and ${ }_{3} \mathrm{C} 58$. The source ${ }_{3} \mathrm{C} 468 \cdot \mathrm{I}$ is two hsiu to the west of $K^{\prime}$ uei and is nearly to deg. from Hua-kai. $3 \mathrm{C} 27^{2}$ is too near Hua-kai to result in two independent references to Ch'uan-shê, a much less well-defined group of stars.

Of the three selected sources, $3 \mathrm{C} 2 \mathrm{I} \cdot \mathrm{I}$ is not in K'uei hsiu but fits the other descriptions fairly satisfactorily. Much of $K^{\prime} u e i$ constellation lies in Tung-pi mansion. ${ }_{3} \mathrm{C}_{\mathrm{II}} \cdot \mathrm{I}$ is also in Tung-pi but it lies very close to Tê's-hsing ( $\kappa$ Cas) and Wang-liang and $6 \mathrm{deg}$. from Hua-kai. This source does not seem to answer the observed locations so well. The remaining source ${ }_{3} \mathrm{C} 58$ lies just within $K^{\prime} u e i$ hsiu. It is $8 \mathrm{deg}$. from Wang-liang but this constellation contains some of the brightest stars 
in Cassiopeia. The proximity to Hua-kai ( $4 \mathrm{deg}$.) is in accord with the Chin account.

The mention of the expression fan in the Sung Shih record is critical. As already seen this is an important astrological term implying a maximum distance of about one degree from a celestial body. The star $\mathrm{BD}+63^{\circ} 99$ (mag. 5.5), lying $2 \cdot 28 \mathrm{deg}$. from $3 \mathrm{C}$ I I $\cdot \mathrm{I}$ and $2 \cdot 15 \mathrm{deg}$. from ${ }_{3} \mathrm{C} 2 \mathrm{I} \cdot \mathrm{I}$, is the nearest star of $C^{\prime}$ 'uan-shê to both of these radio sources. Reference to Table III and the discussion in Section 8 shows that such distances are much too large for the term fan to be applicable. ${ }_{3} \mathrm{C} 58$ lies only $0.68 \mathrm{deg}$. from BD $63^{\circ} 265$ (mag. $5^{\cdot 2}$ ) and $0.53 \mathrm{deg}$. from 53 Cas (mag. 5.6).

Bearing in mind the ample opportunity for observation of the guest star, only ${ }_{3} \mathrm{C} 5^{8}$ can fit all of the evidence from the purely astronomical point of view. The only difficulty in accepting this identification is the lack of reference to Ko-tao. However, this asterism is only rarely mentioned in accounts of both comets and novae (in contrast to Ch'uan-shê). Possibly the explanation is astrological. We read in the Chin Shu (Ho I962, p. 69). 'When it (Ch'uan-shê) is guarded by a guest star, (it warns that) vigilance should be exercised against crafty envoys, and also means that the soldiers of the northern border tribes will take the war-path.' At a time when Mongol and Tartar incursions along the northern border of the Sung empire must have been very frequent, the appearance of a guest star invading Ch'uan-shê for several months would seem most inauspicious to the Sung astronomers.

Very little is known about the radio source ${ }_{3} \mathrm{C} 2 \mathrm{I} \cdot \mathrm{I}$. In the ${ }_{4} \mathrm{C}$ survey, the source $\left(={ }_{4} \mathrm{C} 66.02\right)$ was observed with a flux density of $7.6 \mathrm{f}$.u. at $178 \mathrm{MHz}$. This is only slightly less than the true flux density of $9 \cdot 7 \mathrm{f}$.u., indicating that the angular extent is close to $\mathrm{I}^{\prime} \cdot 5$ arc. The source lies close to the galactic equator $\left(b^{\mathrm{II}}=+4^{\circ}\right)$ and is similar in size to the remnant of Kepler's supernovae. There seems a possibility that $3 \mathrm{C} 2 \mathrm{I} \cdot \mathrm{I}$ is a SNR. However, as already mentioned, the Sung Shih record seems to rule out any connection with the guest star.

${ }_{3} \mathrm{CII} \cdot \mathrm{I}$ ( $={ }_{4} \mathrm{C} \mathrm{63.02}$ ) was observed in the ${ }_{4} \mathrm{C}$ survey without reduction in intensity ( $11 \cdot 6 \mathrm{f}$.u. at $178 \mathrm{MHz}$ ). The source was not resolved in the investigation by Fomalont (I968) at I425 $\mathrm{MHz}$. Fomalont gave the upper limit of the structure diameter as $0^{\prime} \cdot 40$ arc. The spectral indices $\alpha(38 / 750)=-0.78 \pm 0.17$ and $\alpha(750 / 5000)$ $=-0.99 \pm 0.04$ given by Kellermann et al. (1969) are characteristic of a non-thermal source, but the very small size of $3 \mathrm{C}$ II $\mathrm{I}_{\mathrm{I}}$ suggests that it is extra-galactic.

${ }_{3} \mathrm{C} 58\left(={ }_{4} \mathrm{C} 64.02\right)$ has been generally regarded as a galactic source on account of its low galactic latitude $\left(b^{\mathrm{II}}=+3^{\circ}\right)$ and large angular extent since the CTA survey at $960 \mathrm{MHz}$ by Harris \& Roberts (I960). 
In the revised ${ }_{3} \mathrm{C}$ survey the diameter was given as $4^{\prime} \cdot 5 \pm \mathrm{I}^{\prime} \cdot 0$ at $\mathbf{I 7 8}$ $\mathrm{MHz}$. The form measured by Maltby \& Moffett (1962) at $960 \mathrm{MHz}$ was an ellipse, $6^{\prime} \cdot 0 \pm I^{\prime} \cdot 0 \mathrm{E}$.W. and $2^{\prime} \cdot 3 \pm 0^{\prime} 3$ N.S., with some fine structure. The observed flux density in the ${ }_{4} \mathrm{C}$ survey $(7 \cdot 6 \mathrm{f}$.u. at $178 \mathrm{MHz}$ ) was much less than the total flux density (26 f.u.).

The spectrum of ${ }_{3} \mathrm{C} 58$ is very flat. Spectral indices of $a$ ( $\left.178 / 75^{\circ}\right)$ $=+0.1 \mathrm{I} \pm 0.03$ and $a(750 / 5000)=-0.10 \pm 0.03$ were given by Kellermann et al. (1969). Howard et al. (1965a) classed the source as thermal, but subsequent investigators have regarded ${ }_{3} \mathrm{C} 58$ as a probable SNR on account of its high degree of polarization at centimetre wavelengths and low galactic latitude. Sastry et al. (1967) measured the degree of linear polarization at a wavelength of $6 \mathrm{~cm}(5 \mathrm{GHz})$ as $6.7 \pm 0.1$ per cent at a position angle of $166 \pm 1^{\circ}$. At $3.75 \mathrm{~cm}$ wavelength $(8 \mathrm{GHz})$ the corresponding quantities are $5.9 \pm 0.4$ per cent at $175 \pm 4^{\circ}$ (Hobbs \& Haddock, I967). Both results resemble closely those for the Crab Nebula (Tauris A, $3 \mathrm{C}_{144}$ ).

A SNR hypothesis is favoured by the following investigators: Sastry et al. (1967); Kellermann et al. (1968); Pauliny-Toth \& Kellermann (1968); Kellermann et al. (1969); Caswell (1969), (1970). A radio source associated with the guest star of II8I would be twice as old as $3 \mathrm{C} 10$ and ${ }_{3} \mathrm{C} 358$, the remnants of the supernovae of 1572 and 1604, so that an angular extent of several minutes of arc is expected. ${ }_{3} C 58$ seems to qualify well in this respect. Owing to its flat spectrum the intensity of this source at low frequencies is considerably less than that of $3 \mathrm{C}$ 10 or $3 \mathrm{C} 58$, but at high frequencies (in excess of $\mathrm{IGHz}$ ) the intensity is comparable with that of both sources. The integrated flux density of ${ }_{3} \mathrm{C} 5^{8}$ between $38 \mathrm{MHz}$ and $3 \mathrm{GHz}$ given by Howard et al. (1965b) is 9.6 (in units of $10^{-16} \mathrm{~W} \mathrm{~m}^{-2}$ ). This compares with 17.9 for $3 \mathrm{C} 10$ and 6.2 for $3 \mathrm{C}_{358}$. This result suggests that, if identification of ${ }_{3} \mathrm{C} 58$ as the remnants of the quest star is to be made, the star should have been considerably brighter than zero magnitude. However, such a discrepancy could easily be accounted for by visual interstellar absorption; I learn from J.L.Caswell (private communication) that ${ }_{3} \mathrm{C} 58$ lies in a region of heavy optical obscuration.

The agreement between the position of $3 \mathrm{C} 58$ and that of the guest star of II8I is excellent. The 1950.0 position of the radio source is given in $4 \mathrm{C}$ as R.A. $2^{\mathrm{h}} \mathrm{OI}^{\mathrm{m}} 43^{\mathrm{s}} \cdot \mathrm{O}$, Dec. $+64^{\circ} 36^{\prime} 5$. That of the $5^{\circ} \cdot 2$ mag. star BD $63^{\circ} 265$ is $\mathrm{I}^{\mathrm{h}} 55^{\mathrm{m}} 55^{\mathrm{s}} \cdot \mathrm{I}$, $+64^{\circ} 22^{\prime} \cdot 8$ (Smithsonian Catalogue 1966). Proper motion of BD $63^{\circ} 265$ is negligible. I.Rosenberg of the Mullard Radio Astronomy Observatory, University of Cambridge, intends to publish a map of $3 \mathrm{C} 58$ at a frequency of 408 $\mathrm{MHz}$ and with a resolution of $80^{\prime \prime}$ arc. Unless it can definitely be established that the age of the radio source is greater than 2000 years 
(see Section 4), the absence of any other SNR which could be associated with the guest star of 1181 makes the identification of ${ }_{3} \mathrm{C} 58$ as its remnant very convincing.

\section{ACKNOWLEDGMENTS}

I wish to express my gratitude to Mr A.C.Barnes, School of Oriental Studies, University of Durham, for much help with translation of oriental records and for many valuable discussions. My thanks are also due to Mr D.G.Chibbett, School of Oriental and African Studies, University of London, and Mr I.Rosenberg, Mullard Radio Astronomy Observatory, University of Cambridge.

\section{REFERENCES}

Baade, W., 1943. Astrophys. J., 97, I 19.

Baade, W., I945. Astrophys. J., 102, 309.

Bennett, A.S., 1962. Mem. R. astr. Soc., 68, 163.

Caswell, J.L., 1967. Mon. Not. R. astr. Soc., 137, 141.

Caswell, J.L., 1969. Observatory, 89, 230.

Caswell, J.L., 1970. Astr. Astrophys., 7, 59.

Chu Sun-il, 1968. J. Korean astr. Soc., I, 29.

Edge, D.O., Shakeshaft, J.R., McAdam, W.B., Baldwin, J.E. \& Archer, S., 1959. Mem. R. astr. Soc., 68, 37.

Fomalont, E.B., 1968. Astrophys. J. Suppl. Ser., 15, 203.

Gower, J.F.R., Scott, P.F. \& Wills, D., 1967. Mem. R. astr. Soc., 71, 49.

Harris, D.E. \& Roberts, J.A., 1960. Publs astr. Soc. Pacif., 72, 237.

Ho Peng Yoke, 1962. Vistas in Astronomy, Vol. 5, p. 127. Pergamon Press Ltd, Oxford.

Ho Peng Yoke, 1966. The Astronomical Chapters of the Chin Shu. Mouton, Paris.

Hobbs, R.W. \& Haddock, F.T., 1967. Astrophys. J., I47, 908.

Howard, W.E., von Hoerner, S., Aller, H·D. \& Walker, L.R., I965a. Astrophys.J. Suppl. Ser., ro, 323.

Howard, W.E., Dennis, T.R., Maran, S.P. \& Aller, H.D., 1965b. Astrophys. J. Suppl., Ser., 10, 331.

Hsi Tsê-tsung, 1955. Acta Astr. Sinica, 3, 183. Trans. 1958 in Smithson. Contr. Astrophys., 2, 109.

Hsi Tsê-tsung (Xi Ze-zong) \& Po Shu-jen (Bo Shu-ren), 1965. Acta Astr. Sinica, 13, I. Trans. NASA Tech. Trans. TT F-388. Abridged trans. by K.S.Yang, I966, in Science, 154, 597.

Hsüeh Chung-san \& Ou-yang I., 1956. A Sino-Western Calendar for Two Thousand Years, A.D. I-2000. Peking.

Kanda Shigeru, 1935. Nihon Temmon Shiryö (Japanese Astronomical Observations). Tokyo.

Kellermann, K.I., Pauliny-Toth, I.I.K. \& Tyler, W.C., 1968. Astr. J., 73, 298.

Kellermann, K.I., Pauliny-Toth, I.I.K. \& Williams, P.J.S., I969. Astrophys. J., I57, I.

Knobel, E.B., 1909. Mon. Not. R. astr. Soc., 69, 435.

Maltby, P. \& Moffet, A.T., 1962. Astrophys. J. Suppl. Ser., 7, 14 I.

Minkowski, R., I968. Nebulae and Interstellar Matter, Chapter 11. Eds B.M. Middlehurst \& L.H.Aller, University of Chicago Press. 
Needham, J., 1959. Science and Civilisation in China, Vol. 3. Cambridge University Press.

Newcomb, S., 1895. Tables of the Sun and Inner Planets, Astr. Pap. Amer. Eph., 6.

Pauliny-Toth, I.I.K. \& Kellermann, K.I., I968. Astr. J., 73, 953.

Payne-Gaposchkin, C., 1956. The Galactic Novae. North Holland Publishing Co., Amsterdam.

Rigge, W.F., I91 5. Popular Astr., 23, 29.

Rufus, W.C., I913. Trans. Korea Br. R. Asiatic Soc., 4, 23.

Rufus, W.C., I915. Popular Astr., 23, 193.

Rufus, W.C. \& Chao, C., 1944. Isis, 35, 316.

Rufus, W.C. \& Tien Hsing-chih, 1945. The Soochow Astronomical Chart. University of Michigan Press, Ann Arbor.

Sastry, C.V., Pauliny-Toth, I.I.K. \& Kellermann, K.I., 1967. Astr. J., 72, 230.

Schlegel, G., 1875. Uranographie Chinoise. Brill, Leyden.

Schlesinger, F. \& Jenkins, L.F., I940. Catalog of Bright Stars. Yale University Observatory, New Haven.

Shakeshaft, J.R., Ryle, M., Baldwin, J.E., Elsmore, B. \& Thomson, J.H., 1955. Mem. R. astr. Soc., 67, 106.

Shinoda Minoru, I960. The Founding of the Kamakura Shogunate II80-II85, pp. 220-21. Columbia University Press, New York.

Shklovsky, I.S., 1960. Cosmic Radio Waves, pp. 280-81. Trans. R.B.Rodman \& C.M.Varsavsky, Harvard University Press, Cambridge, Mass.

Smithsonian Astrophysical Observatory, 1966. Star Catalog. Smithsonian Institution, Washington, D.C.

Tuckerman, B., 1964. Planetary, Lunar and Solar Positions, A.D. 2 to A.D. I649, Mem. Am. phil. Soc., 59.

Wylie, A., I897. Chinese Researches, Shanghai.

Zwicky, F., 1965. Stellar Structure, Chapter 7. Eds L.H.Aller \& D.B.McLaughlin, University of Chicago Press. 